\title{
Atrial Pacing May Decrease the Incidence of Atrial Fibrillation Following Coronary Artery Bypass Graft Surgery
}

M Cohen*, JF Morin ${ }^{Y}$, M.D., and M Rosengarten ${ }^{Y}$, M.D.

* To whom correspondence should be addressed: Faculty of Medicine, McGill University, 3655 Drummond St., Montreal, QC, Canada H3G 1Y6

Ý Department of Cardiovascular and Thoracic Surgery, Montreal General Hospital, McGill University, 1650 Cedar Avenue, Montreal, QC, Canada H3G 1A4

\section{ABSTRACT}

At the Montreal General Hospital in 1994, the incidence of atrial fibrillation (AF) following coronary artery bypass graft (CABG) surgery was 30\%. With the goal of reducing the incidence of this complication, 20 patients (15 males and 5 females) undergoing CABG surgery were studied prospectively for the occurrence of AF following a three-day "on demand" atrial pacing at 90 beatsper-minute. The age range of the group was 39 to 79 years, with a mean age of 60 and a median age of 63. Two patients were categorized in NYHA class II, 13 in class III, and five in class IV. Of the latter five, two patients received intravenous nitroglycerin preoperatively. B-blockers were taken preoperatively by 13 patients, $\mathrm{Ca}^{2+}$ channel blockers by 12 , and $\mathrm{ACE}$ inhibitors by two. Only one patient had suffered a myocardial infarction within the last year. Preoperative ejection fractions ranged from 15 to $60 \%$ with a mean of $45 \%$. In all cases, the CABG operation achieved complete revascularization using left internal mammary artery (LIMA) grafts in addition to saphenous vein grafts. The average number of bypasses performed was 3.15. Cardiac arrest was induced using intermittent cold blood cardioplegia, and cardiopulmonary bypass time averaged 60 minutes. Aortic occlusion time averaged 36 minutes. Intraoperative inotrope use was infrequent, used intraoperatively with two cases requiring neosynephrine, one case requiring levophed, and one case requiring dobutamine. Post-pacing cardiac assessment revealed non-specific $T$ wave abnormalities in five $(25 \%)$ of patients, but no patient showed any evidence of acute myocardial infarction. In no cases did any complications related to the atrial pacing develop in the postoperative period. The results reveal that only two patients (15\%) experienced AF following, and one during, the three-day regimen of atrial pacing. Thus, this study provides preliminary evidence of a decrease in the incidence of post-CABG AF using atrial pacing. 
Atrial fibrillation and flutter (AF) are among the most common complications of coronary artery bypass graft (CABG) surgery, with a reported incidence ranging from 10 to $40 \%$ (1-6). At the Montreal General Hospital, the incidence was $30 \%$ in 1994 . While AF is rarely a cause of major morbidity, it can contribute significantly to patient discomfort and may be responsible for prolonged postoperative convalescence.

The symptoms and systemic effects of AF result from an alteration in the normal ability to regulate cardiac rate, rhythm, and atrioventricular contractile sequence in response to the body's needs (7). During AF, multiple overlapping waves of reentrant electrical activity produce an excessively rapid, chaotic atrial rhythm, resulting in rates of atrial contraction greater than 350/min. Consequently, patients in acute AF may experience a variety of symptoms ranging from palpitations (accompanied by chest discomfort, weakness, dizziness, and breathlessness), to progression of ischemic syndromes (secondary to increased myocardial oxygen requirements), to hemodynamic collapse and sudden death. In the absence of effective anticoagulation therapy, patients with sustained AF may also be susceptible to the development of systemic emboli resulting from blood stasis and subsequent clot formation in a functionally non-contractile atrium. However, the risk of embolism in AF depends on the latter's etiology, and any decision to anticoagulate must balance potential risks and benefits (8).

Various risk factors for the development of post-CABG AF have been postulated. These include advancing age, atrial enlargement, and withdrawal from preoperative $\beta$-blocker therapy $(9,10,13)$. Myocardial factors such as the degree of protection from ischemia afforded by intraoperative cardioplegia are also likely to play a role (9). Unfortunately, none of these predictors has been clearly linked to postoperative AF (10) and, in general, the underlying pathogenesis of post-CABG AF remains elusive and may be multifactorial (11).

Similarly, there is little agreement on suitable prophylaxis for post-CABG atrial arrhythmias $(12,13)$. Many trials have examined the utility of various pharmacologic methods in the prevention of postoperative AF. Reports of pharmacologic effectiveness have been highly variable and, more significantly, the adverse effects of these agents have provided an argument against their routine use for prophylaxis (11). Other studies have proposed that non-pharmacologic methods, such as cardiac pacing, may be effective in preventing atrial arrhythmias $(14,15)$. The purpose of this study is to provide a preliminary investigation of the efficacy of atrial pacing as a means of preventing post-CABG atrial arrhythmias.

\section{MATERIALS AND METHODS}

In this pilot study, 20 consecutive patients undergoing CABG surgery at the Montreal General Hospital were studied for the occurrence of AF following a three-day regimen of "on demand" fast-rate atrial pacing. The "on demand" atrial pacing regimen employs a pacemaker unit that senses electrical activity in the heart and fires, through epicardial pacing wires only when the patient's heart rate falls below a set value. Of these patients, 15 were male and five were female. Subjects ranged from 39 to 79 years of age, with a mean age of 60 and a median age of 63 .

\section{Preoperative Assessment}

Patients were categorized according to the New York Heart Association classification (Class I: angina after heavy excercise; Class II: angina after walking; Class III: angina while talking/breathing; Class IV: angina at rest/on waking. Two patients were designated NYHA class II, 13 designated class III, and five designated class IV. Of the latter five patients, two received intravenous nitroglycerin preoperatively. Preoperative ejection fractions (EF) were also measured and ranged from 15 to $60 \%$, with a mean of $45 \%$. Preoperative use of cardiac medications was frequently encountered, with $\beta$-blockers taken by 13 patients, $\mathrm{Ca}^{2+}$-channel blockers by 12 , and ACE inhibitors by two. Only one patient had suffered a documented myocardial infarction within one year prior to surgery. 


\section{Surgical Technique}

In all cases, the $\mathrm{CABG}$ operation achieved complete revascularization using left internal mammary artery (LIMA) grafts in addition to saphenous vein grafts. On average, 3.15 bypasses were performed per patient. Cardiac arrest was induced using intermittent hypothermic cardioplegia, and cardiopulmonary bypass time averaged 60 minutes. Aortic occlusion time averaged 36 minutes. Inotropes were used intraoperatively in only four patients, with two cases requiring neosynephrine, one requiring levophed, and the last requiring dobutamine.

\section{Postoperative Pacing}

Postoperatively, all patients were followed regularly by the cardiac surgery team in the surgical intensive care unit and, later, on the cardiac surgery wards. Atrial pacing was initiated immediately post-CABG and was continued for three days at a fixed rate of 90 beats-per-minute (bpm). Post-pacing cardiac assessment was by ECG and serum creatinine kinase $(\mathrm{CK})$ measurement.

\section{RESULTS}

After three consecutive days of post-CABG atrial pacing, ECG revealed non-specific T-wave abnormalities in five $(25 \%)$ patients. No patients showed any evidence of acute myocardial infarction. Serum CK measurements ranged from 221 to 1651 with a mean of 498 and a median of 334. With one exception, all patients resumed B-blocker therapy on the first day following CABG. Only three (15\%) patients experienced AF, two following the three-day regimen of atrial pacing and the third, who had not resumed B-blocker therapy early after surgery, during pacing.

\section{DISCUSSION}

Arrhythmia following CABG surgery is a common problem, and shows an increased prevalence among elderly patients (10). While AF is usually benign and self-limiting, it may be associated with hemodynamic instability, prolonged hospitalization, and embolic stroke (12). Because these complications can be particularly deleterious in an increasingly elderly patient population, there is clearly a need for the development of effective prophylaxis against postoperative atrial arrhythmias.

In general, the treatment of AF is directed toward the management of any underlying disorder, the conversion of AF to sinus rhythm (and maintenance of this rhythm after conversion), the control of ventricular response rate when $\mathrm{AF}$ is not converted, and the prevention of emboli. To date, most research into the prevention of post-CABG AF has focused on the use of pharmacologic methods, including digoxin, B-blockers, magnesium sulfate, and amiodarone, with frequently contradictory results.

Postoperative digoxin has been recommended as an effective prophylaxis against postoperative $\mathrm{AF}$ in some studies $(1,3,16-18)$ but has been rejected in others (2). Typically, digoxin itself is highly effective at controlling cardiac frequency in rapid $\mathrm{AF}$, but must be combined with a $\beta$-blocker or $\mathrm{Ca}^{2+}$-channel blocker when there is a decrease in vagal tone and an increase in sympathetic activity (8). The use of B-blockers, such as sotalol, as single-drug prophylaxis against AF has been investigated in various studies. One such study showed that sotalol was effective in reducing the incidence of AF in spite of concerns regarding the side effects of ß-blockade (19). Conversely, another study showed no significant difference in the incidence of AF between sotalol patients and controls (20). Moreover, it has been suggested that the reduced effectiveness of postoperative $\beta$-blockers in elderly patients may make its routine postoperative use inappropriate (5). As is the case for ß-blockers, the prophylactic use of magnesium sulfate has been shown to reduce both the incidence and severity of arrhythmias post-CABG in some studies $(21,22)$, but not in another (23). Finally, although amiodarone therapy has been shown to prevent clinically-significant arrhythmias, reports of 
bradycardia following its postoperative administration may limit its usefulness (4). Thus, despite the potential utility of pharmacotherapy in the preventiion of post-CABG arrhythmia, trials to date have shown highly variable results. As such, no effective prophylactic regimen has yet been established $(12,13)$ and treatment of AF remains controversial and often problematic (7).

In this study, fast-rate atrial pacing was investigated as an alternative to pharmacologic management. The premise of this work is based on clinical reports suggesting that the atrium in non-surgical patients with sick sinus syndrome is less likely to develop AF (14), and on studies suggesting that pacing may be beneficial in preventing episodes of paroxysmal AF (15). A similar logic has prompted the present investigation of the benefits of atrial pacing in the prophylaxis of arrhythmia in post-CABG patients.

Of the 20 patients prophylaxed with atrial pacing for three consecutive days postoperatively, only three developed AF. One of these three patients had not resumed preoperative B-blocker use postoperatively, providing a likely explanation for this occurence of AF. The two remaining cases of AF occurred after cessation of the three-day pacing regimen.

In future trials, a four-day atrial pacing regimen will be adopted, considering that the peak incidence of arrhythmia following cardiac surgery occurs on the second or third postoperative day in non-paced patients (13). In addition, atrial pacing will be augmented to $100 \mathrm{bpm}$ in order to overdrive the atrium while respecting the limits of normal myocardial function. This elevated pacing rate is likely to permit greater control over atrial activity without inducing myocardial edema, and thus may extend the benefits of postoperative atrial pacing to an expanded patient population.

Overall, a $15 \%$ incidence of post-CABG AF is found, representing a 50\% decrease in the incidence of this complication as reported at the Montreal General Hospital. However, a more extensive prospective casecontrol study is needed to further investigate these highly-encouraging preliminary results.

Due to the paucity of data on postoperative AF and to the absence of a gold standard for its prediction (23), future trials will emphasize extensive preoperative, perioperative, and postoperative data. These trials will institute Holter monitoring to record cardiac electrical activity and thus confirm proper functioning of the pacemaker. Cardiac output, heart rate, blood pressures, and wedge pressures will be carefully monitored to ensure that the pacing regimen does not cause myocardial damage or induce heart failure. Future trials will also implement the following investigations: thyroid function tests to identify and exclude patients who may have increased risk of AF due to hyperthyroidism; echocardiography to measure left atrial size, a postulated risk for post-CABG AF; and analyses of ventricular arrhythmias. An attempt will also be made to identify specific risk factors for post-CABG AF, as the causes of AF have not yet been elucidated satisfactorily (25). Through further collection and analysis of pertinent data, future investigations promise to clarify the significance of the current finding that atrial pacing provides considerable benefit in preventing post-CABG atrial arrhythmias.

\section{REFERENCES}

1. Mohr R, Smolinsky A, Goor DA. Prevention of supraventricular tachyarrhythmia with low-dose propanolol after coronary bypass. Journal of Thoracic \& Cardiovascular Surgery 81(6): 840-845; 1981.

2. Ivey MF, Ivey TD, Bailey WW, et al. Influence of propanolol on supraventricular tachycardia early after coronary artery revascularization. A randomized trial. Journal of Thoracic \& Cardiovascular Surgery 85(2): 214-218; 1983.

3. Matangi MF, Neutze JM, Graham KJ, et al. Arrhythmia prophylaxis after aorta-coronary bypass. The effect of minidose propanolol. Journal of Thoracic \& Cardiovascular Surgery 89(3): 439-443; 1985. 
4. Butler J, Harriss DR, Sinclair M, Westaby S. Amiodarone prophylaxis for tachycardias after coronary artery surgery: a randomised, double blind, placebo controlled trial. British Heart Journal 70(1): 56-60; 1993.

5. Fuller JA, Adams GG, Buxton B. Atrial fibrillation after coronary artery bypass grafting. Is it a disorder of the elderly? Journal of Thoracic \& Cardiovascular Surgery 97(6): 821-825; 1989.

6. Butler J, Chong JL, Rocker GM, et al. Atrial fibrillation after coronary artery bypass grafting: a comparison of cardioplegia versus intermittent aortic cross-clamping. European Journal of Cardio-Thoracic Surgery 7(1): 23-25; 1993.

7. Nattel S. Newer developments in the management of atrial fibrillation. American Heart Journal 130(5): 1094-1106; 1995.

8. Thumala A. [When, why, and how to treat atrial fibrillation]. Revista Medica de Chile 123(1): 90-97; 1995.

9. Mendes La, Connelly GP, McKenney PA, et al. Right coronary artery stenosis: an independent predictor of atrial fibrillation after coronary artery bypass surgery. Journal of the American College of Cardiology 25(1): 198-202; 1995.

10. Leitch JW, Thomson D, Baird DK, Harris PJ. The importance of age as a predictor of atrial fibrillation and flutter after coronary artery bypass grafting. Journal of Thoracic \& Cardiovascular Surgery 100(3): 33842; 1990 .

11. Saloman N. Atrial fibrillation following coronary artery bypass surgery: new hope for an old problem? Critical Care Medicine 21(10): 1421-1422; 1993.

12. Terada Y, Mitsui T, Yamaguchi I. Conduction disturbances after open heart operation in a patient receiving amiodarone. Annals of Thoracic Surgery 58 (4): 1213-1214; 1994.

13. Frost L, Molgaard H, Christiansen EH, et al. Atrial fibrillation and flutter after coronary artery bypass surgery: epidemiology, risk factors, and preventive trials. International Journal of Cardiology 36(3): 253-261; 1992.

14. Lamas GA, Estes NM 3d, Schneller S, Flaker GC. Does dual chamber or atrial pacing prevent atrial fibrillation? The need for a randomized controlled trial. Pacing \& Clinical Electrophysiology 15(8): 1109$1113 ; 1992$.

15. Glikson M, Espinosa RE, Hayes DL. Expanding indications for permanent pacemakers. Annals of Internal Medicine 123(6): 443-451; 1995.

16. Stephenson LW, MacVaugh H 3d, Tomasello DN, Josephson ME. Propanolol for prevention of postoperative cardiac arrhythmias: A randomized study. Annals of Thoracic Surgery 29(2): 113-116; 1980.

17. Ormerod OJ, McGregor CG, Stone DL, et al. Arrhythmias after coronary bypass surgery. British Heart Journal 51(6): 618-621; 1984.

18. Rubin DA, Nieminski KE, Reed GE, Herman MV. Predictors, prevention, and long-term prognosis of atrial fibrillation after coronary artery bypass graft operations. Journal of Thoracic \& Cardiovascular Surgery 94(3): 331-335; 1987.

19. Nystrom U, Edvardsson N, Berggren $\mathrm{H}$, et al. Oral sotalol reduces the incidence of atrial fibrillation after coronary artery bypass surgery. Thoracic \& Cardiovascular Surgeon 41(1): 34-37; 1993. 
20. Jacquet L, Evenepoel M, Marenne F, et al. Hemodynamic effects and safety of sotalol in the prevention of supraventricular arrhythmias after coronary artery bypass surgery. Journal of Cardiothoracic \& Vascular Anesthesia 8(4): 431-436; 1994.

21. Fanning WJ, Thomas CS Jr, Roach A, et al. Prophylaxis of atrial fibrillation with magnesium sulfate after coronary artery bypass grafting. Annals of Thoracic Surgery 52(3): 529-353; 1991.

22. Casthely PA, Yoganathan T, Komer C, Kelly M. Magnesium and arrhythmias after coronary artery bypass surgery. Journal of Cardiothoracic and Vascular Anesthesia 8(2): 188-191; 1994.

23. Parikka H, Toivonen L, Pellinen T, et al. The influence of intravenous magnesium sulphate on the occurrence of atrial fibrillation after coronary artery bypass operation. European Heart Journal 14(2): 251$825 ; 1993$.

24. Klein M, Evans SJ, Blumberg S, et al. Use of P-wave-triggered, P-wave signal-averaged electrocardiogram to predict atrial fibrillation after coronary artery bypass surgery. American Heart Journal 129(5): 895-901; 1995.

25. Frost L, Jacobsen CJ, Christiansen EH, et al. Hemodynamic predictors of atrial fibrillation or flutter after coronary artery bypass grafting. Acta Anaesthesiologica Scandinavica 39(5): 690-697; 1995.

\section{BIOGRAPHY}

Matthew G. Cohen is presently a third-year medical student at McGill University (Montreal, Quebec, Canada). His research on post-CABG atrial pacing was conducted at McGill during the present year, in conjunction with the Departments of Cardiology and Cardiovascular and Thoracic Surgery at the Montreal General Hospital. He is currently involved in the research of alternate treatment strategies for late vascular graft infections. He will continue his research on post-CABG atrial fibrillation prophylaxis in the Spring of 1996 and eventually plans to pursue a surgical residency.

Copyright (C) 1996 by MJM 